Case Report

\title{
Fabrication of a Silicone Auricular Prosthesis - A Case Report
}

\author{
Krishna Prasad D. ${ }^{1}$, Archana Ashok Swaminathan ${ }^{2} \&$ Anupama Prasad D. ${ }^{3}$
}

${ }^{1}$ Professor \& HOD, ${ }^{2}$ Post Graduate, ${ }^{3}$ Lecturer, Department of Prosthodontics and Crown \& Bridge,

A.B. Shetty M emorial Institute of Dental Sciences, Nitte University, M angalore, Karnataka, India.

\author{
Correspondence \\ Krishna Prasad D. \\ Professor \& HOD, Department of Prosthodontics and Crown \& Bridge, \\ A.B. Shetty M emorial Institute of Dental Sciences, Nitte University, M angalore - 575 018, Karnataka, India. \\ E-mail : drkrishnaprasadd@yahoo.in
}

\begin{abstract}
The rehabilitation of a patient with facial defects is a challenging task. An alternative to surgical reconstruction is the creation of a silicone auricular prosthesis. The replacement of anatomical parts is an art and science. These prostheses provide a cost-effective and an acceptable means of camouflage for patients who are disinclined to surgical reconstruction. The process of creating a silicone auricular prosthesis is discussed in this article.
\end{abstract}

Keywords: Ear prosthesis, Auricular prosthesis, silicone prosthesis, maxillofacial prosthesis

\section{Introduction}

M an's need for beauty has probably existed as long as man himself. Artificial replacements to replace lost body parts have been documented well even from ancient times. Body abnormalities or defects compromise appearance, a factor that renders an individual incapable of leading a relatively normal life. An alternative to surgical reconstruction is the creation of a silicone auricular prosthesis. These prostheses provide a cost-effective and cosmetically acceptable means of camouflage for patients who decline orpostpone surgical reconstruction.[1]

The replacement of anatomical parts is an art and science. Prosthesis form, coloration, texture must be as indiscernible as possible from the surrounding natural tissue. The ideally constructed prosthesis must duplicate the missing facial features so precisely that the casual observer notices nothing that would draw attention to the prosthetic reconstruction.

\begin{tabular}{|c|}
\hline Access this article online \\
\hline Quick Response Code \\
\hline
\end{tabular}

The primary objective of maxillofacial prosthetics is to restore esthetics, function and preserve the remaining hard and soft t i s s u e s. The accomplishment of the primary objective often leads to the important secondary objective of restoring the individual to the society and enabling them to lead a normallife.

There are several advantages to silicone maxillofacial prosthesis. It requires little surgery or no surgery, the patient spends less time away from home and job and the reconstruction is often more natural-looking. However, the drawbacks include the necessity of fastening the appliance to the skin and removing it every day. The function of the prosthetic ear is to direct the sound waves into the auditory canal and to maintain a proper environment for the inner ear membranes. It normally improves hearing by about $20 \%$.The prosthetic ear will retain eyeglasses, and retain a hearing aid if needed. It also serves as a great psychological benefit in the rehabilitation of the patient.

\section{Steps in Fabrication}

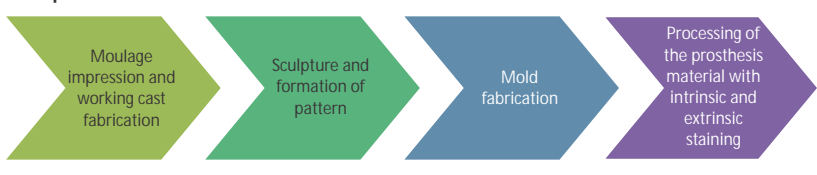

\section{Case Report}

The patient, aged 23 years, came to the Department of Prosthodontics, complaining of missing ear on the left side. On examination it was he was found to have a normal ear on right side with normal hearing. A small remnant of ear was present on left side with no auditory canal opening was 
seen. The patient claimed to have hearing capacity of about $20 \%$ on the left side. The patient was diagnosed to have microtia of the left side of the ear. (Fig 1 )

Treatment Plan

The entire treatment was divided into four appointments.

- Impressions

- Fabricating waxpattern

- Makingthe mould

- Processing the prosthesis

To make an impression, the patient's head was tilted with the auricular area as horizontal as possible. Petroleum jelly was applied to the hair surrounding the area and the external auditory meatus was blocked with a cotton pellet.

A cylindrical cardboard cover was modified as a tray to fit the patients right ear, and the remnant on the left side.(Fig 2) Relief holes were made in this receptacle with a no. 6 round bur. A fluid mix of alginate was made, loaded in a syringe and injected under the helix, so as to provide support for the helix when full amount of alginate is added. Remaining material was loaded and the tray was held in position against the patient's ear. (Fig 3)Care should be taken so as not to compress the ear. A wet gauge was placed over it and dental stone poured over the set alginate, to add the support for the alginate in the tray.

The impression was inspected. There should not be any voids or bubbles on the impression. Type IV dental stone was mixed according to the manufacturer's instruction and the impressionswere poured.

\section{Wax pattern fabrication}

Once the impressions were cast, the model of normal ear will provide the necessary landmarks for defective ear around which the carving can begin. The carving detail of the ear is commenced, during which the size is constantly checked with normal ear model. Better results are obtained if the ear is carved from a mirror image of the patient's natural ear. The projection of the ear is measured to achieve the correct distance. Using a rolled length of wax, helixis then added. (Fig 4)
Tryin

The following points are checked at try in. (Fig 5)

- The fit of the prosthesis on the tissue

- The correct horizontal alignment with the natural ear.

- The projection of the ear in relation to the side of the head

- The integrity of the margins

\section{Investment and fabrication of mould}

The wax prosthesis is now sealed to the model and the leading edge is thinned as much as possible so as to allow the silicone edges to feather into the natural skin.[2]

A three part mould is necessary to achieve easy placement of silicone. Embed the mould in plaster up to the leading edge. The middle section of the flask is added and stone is filled into the entire undercut section of the mould along the part line. After a suitable separating medium is applied, the remainder of the flask is filled with stone and is closed. Also, the plaster can be soaked in soap solution which acts as a separator. The helixundercut is poured in a hard dental stone. When pouring the section, finish the plaster so that the flash line will be on the undercut side of the helix. Allow to set, and then cut grooves to allow location with the top half of the mould. M ould is again soaked in soap solution.

When the wax is boiled out of the flask, the three piece mould is left behind. (Fig 6)

\section{Technical considerations}

Cold mould seal, although an excellent method of mould separation, can present as a problem when using silicone. If it is applied too thick, it may lift away from the plaster and get trapped in the silicone.

An added problem is that it can cause the silicone to slide on the surface of the mould. In order to overcome these problems thin down the cold mould seal with water by 50 $\%$. The dry plaster mould is then coated with it and immediately rinsed with running water. The excess is blown away using an airline. The whole mould is left to dry thoroughly. 


\section{Processing of the prosthesis}

Uncolored silicon and liquid catalyst is weighed on weighing machine. This desired amount is mixed properly on a clean dry clear glass slab to avoid air entrapment Improper mixing will lead to porosity

M ixing color for facial prosthesis is a trial and error process of adding pigments in small quantities to silicone and frequently comparing the mixture to patient's skin. The mould cavity is prepared by coating the external tissue surface area with a thin coat of catalyzed uncolored silicone material. Characterization colors are chosen and mixed with silicone polymer and painted on the surface of the clear layer. After the mould surface is characterized by localized application of color, a base color mixture of silicone material is prepared to fill the mould cavity.(Fig 7)Colored rayon fibers may be sprinkled into it to simulate microvasculature

The mould is then clamped and placed into dry heat oven at the manufacturers prescribed polymerization time and temperature.

Residual silicone may be left on the external surface of the mould to test for complete polymerization. After the polymerization cycle is complete, the mould should be allowed to cool to room temperature before removing the completed prosthesis.

\section{ColouringTechniques}

\section{Intrinsic coloration}

Intrinsic coloration is color applied within the mould during the casting procedure.

\section{Extrinsic coloration}

Extrinsic coloration is color applied to the surface of a prosthesis that has been cured and removed from the mould.

Creating a facial prosthesis that appears to have a realistic skin surface while achieving seamless visual integration with the surrounding tissue requires both artistic and technical expertise. A prosthesis that is too light in value can be corrected with extrinsic coloration; however, a prosthesis that appears too dark in value is difficult to remedy and most often needs to be reprocessed.

Extrinsic coloring is necessary to disguise the appearance of a seam along the helix and to blend the anterior margin to the surrounding tissues. However the application of the extrinsic glazes often results in a glossy appearance that many appear unnatural and draw unwanted attention to the prosthesis

\section{Lighting considerations}

If color corrected lighting is not available, incandescent with ample natural light may be used. A color match is best evaluated under various light sources such as day light, fluorescent and incandescent to reduce metamerism.

\section{Identifyingand mixing the base color}

The base color makes up the bulk of the prosthesis; therefore, mix ample material to fill the mould. Considering that the skin is laminar, the objective is to mix the color of the underlying skin tone. One of the most common approaches to mixing color for facial prosthesis is a trial and error process of adding pigments in small quantities to silicone and frequently comparing the mixture to patient's skin in adequately lit environment.

Common places to identify the base color are on the underside of the fore arm, along the hairline, anterior to the tragus, and at base of the helix. (Fig 8)

For repeatability, pigments amount added should be measured and recorded by weight or dropper. When the color of the silicone approaches the desired base color, place a small amount of colored silicone in the center of a folded transparent sheet. Compare the mixed silicone color with the target base color of the patient's skin and modify as necessary.

\section{Surface characterization}

Incorporation of surface characterization is important in creating lifelike results. Freckles, moles, broken capillaries, prominent blood vessels and other skin markings on the contra lateral ear can be used to characterize the prosthesis. 


\section{nUHS}

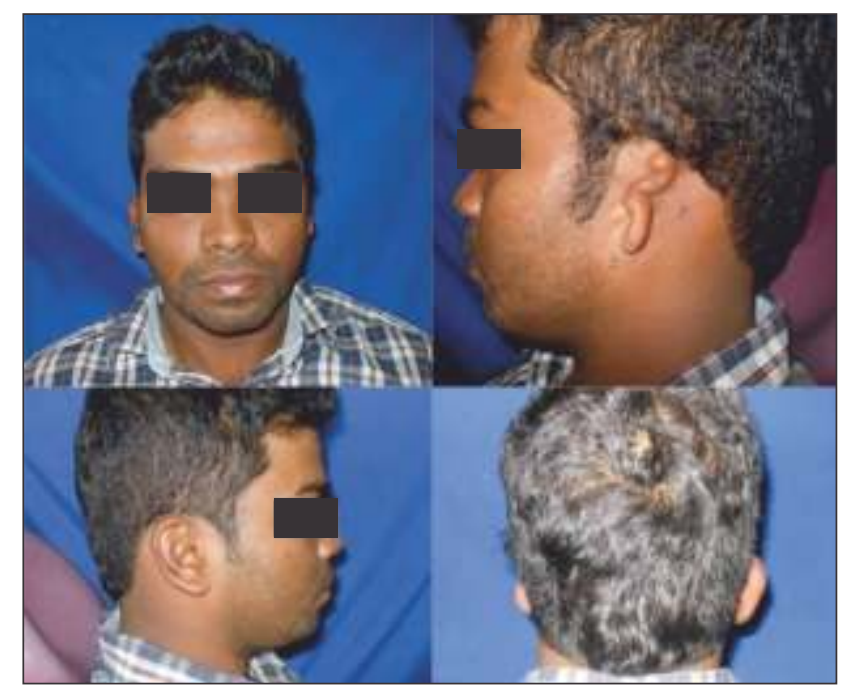

Fig. 1 : Clinical presentation

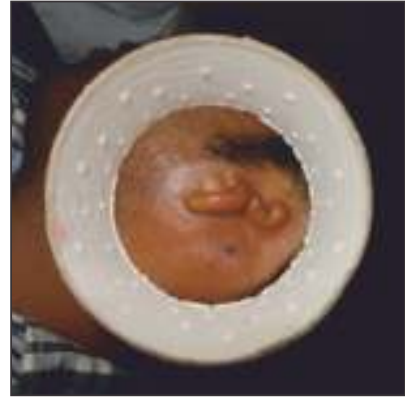

Fig. 2 : Cardboard tray

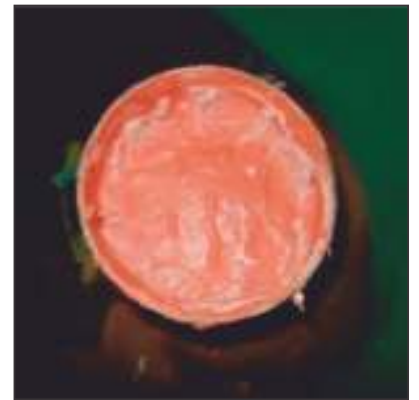

Fig. 3 : Impression

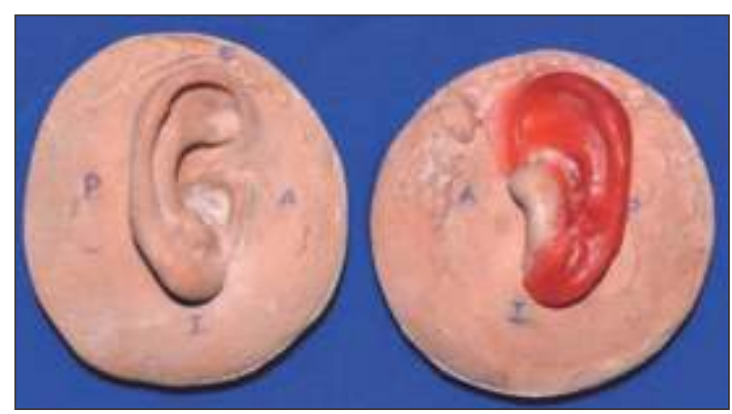

Fig. 4 : Wax up

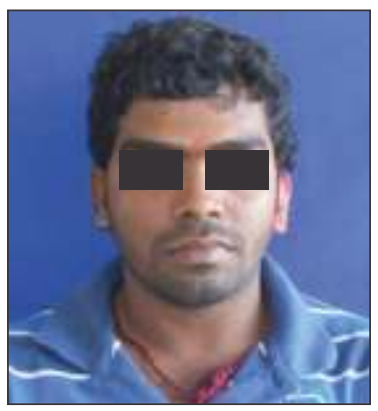

Fig. 5 : Trial

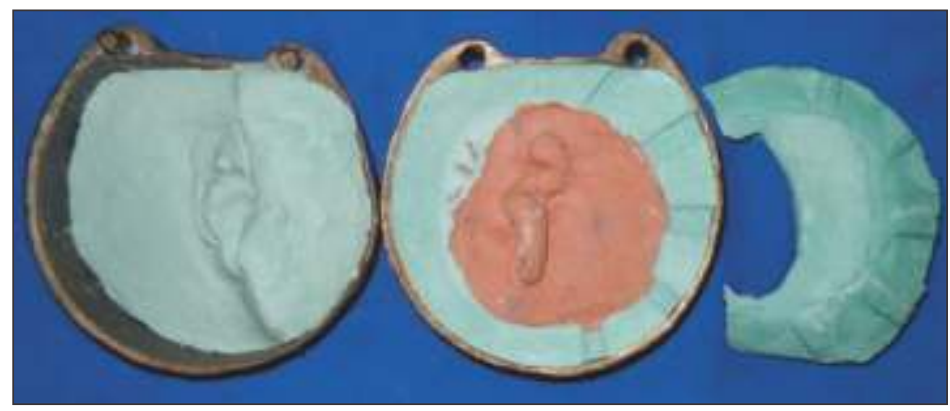

Fig. 6 : Three piece mould

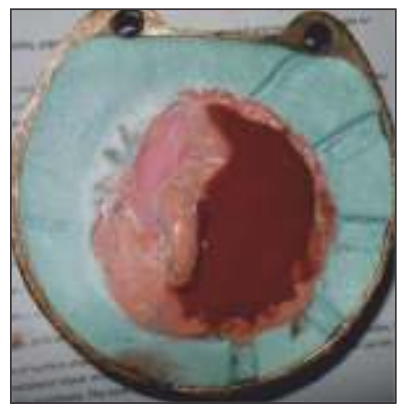

Fig 7: Packing of silicone

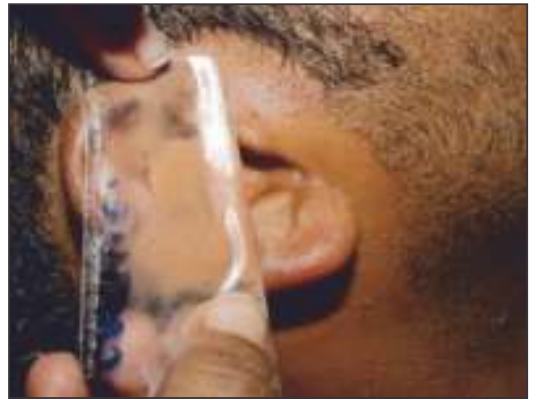

Fig 8: Shade matching

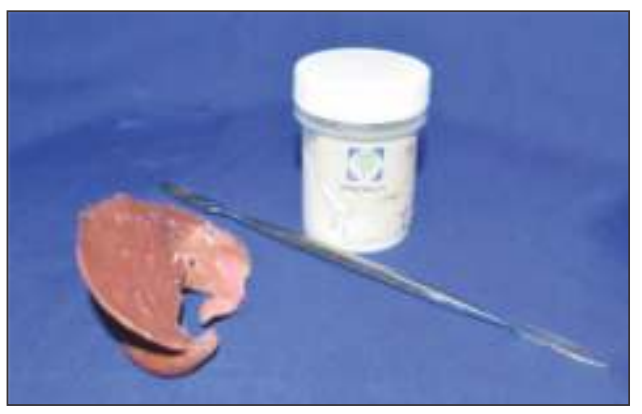

Fig 9: Adhesive
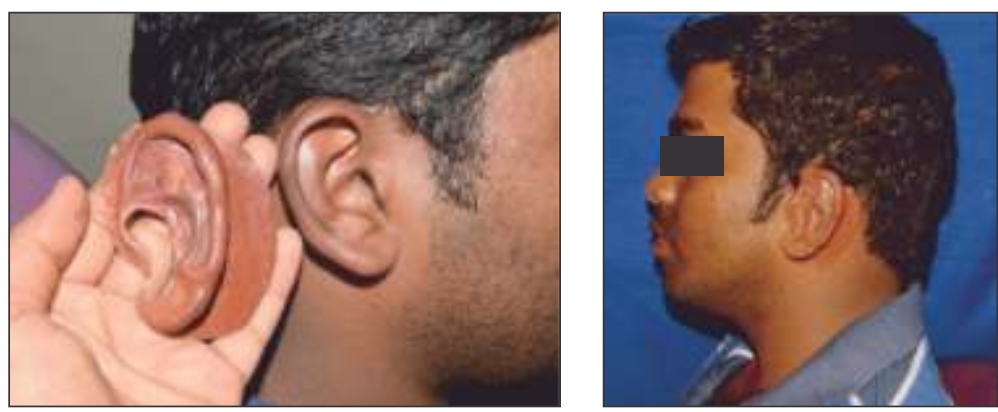

Fig. 10 : Fit of prosthesis 


\section{Skin adhesives}

A variety of adhesive systems have been employed to retain facial prosthesis in position. They are mainly classified by the method in which they are dispensed: pastes, liquid, emulsions, spray, and double sided tapes. Most facial prostheses are retained with medical grade adhesives.

\section{Fit of prosthesis}

When the desired intrinsic shade of a skin was obtained, the auricular prosthesis was tried in patient to check for accuracy of shade and color of the skin on contra lateral side of the face. Once the desired skin shade was obtained, the extrinsic stains (Cosmosil) were painted on prosthesis to match the skin shade on contra lateral side of the face.(Fig 9) Dry air was blown over the prosthesis with the help of a dry air syringe to cure the extrinsic stain. The prosthesis was tried in. Few adjustments and minor modifications were done for better retention and marginal adaptation of the prosthesis. The patient was informed about the limitation and retention aspect of the prosthesis.

Satisfactory retention and stability was achieved by using skin adhesive (Beta Bond, M edical Graded Adhesives) and anatomical and soft tissue undercuts. (Fig 10)

The patient was instructed to apply the skin adhesive over the defect area and leave it over for 2 minutes, so that the adhesive becomes more transparent and then to place the auricular prosthesis over the defect. The frame of the eyeglass can be used to gain additional retention and stability.

The patient was instructed about the use and follow up care of the ear prosthesis.

\section{Discussion}

There are two main reasons for auricular defects Congenital and Acquired.

Congenital involves abnormalities of the first and second branchial arches. Congenital anomalies manifests as microtia or anotia. In mircotia, there is atresia of external auditory meatus and the remnant is 'comma' shaped with upper portion containing a small nubbin of deformed cartilage. Anotia is complete absence of the auricle and is completely rare. Congenital anomalies occur in concurrence with syndromes such as Treacher Collins syndrome and Goldenhaar's syndrome.[3]

Acquired causes could be trauma, burns or malignancies. The more common malignancies include squamous cell carcinoma, basal cell carcinoma and malignant melanoma. The most specific and recommended treatment modality for large tumours and malignancies in the head and neck region is by surgical excision, with or without chemotherapy. After surgical removal, it has to be restored with silicone prosthesis. Hence the Prosthodontist plays major role in fabricating and rehabilitating such large defects on face, occurred by performing radical maxillofacial oncosurgery

\section{Surgical reconstruction versus prosthetic restoration}

The choice between surgical and prosthetic rehabilitation of large defects is a complex decision depending on size and etiology of the defect as well as on wishes of the patient. Basis of major ear reconstruction is the fabrication of a framework from rib cartilage held with stainless steel wire and buried under thin skin. The construction of facial prostheses consists of four stages, each equally important to the success of rehabilitation effort and each requiring extraordinary attention in detail and is a challenge. [4]

\section{Difficulties}

The difficulties faced during fabrication of custom made prosthesis are; obtaining accurate impression of the defect without any compression or distortion of tissue, orientation of ear in harmony with the contra lateral ear, sculpturing the exact anatomy and position of the prosthesis, obtaining a satisfactory shade exactly matching to the skin complexion of contra lateral side of the face.

The location of the prosthetic auricle is predetermined by first observing the topographic relationship of opposite normal ear with facial features in cases of unilateral prosthetic reconstruction and then duplicating its position at the proposed reconstruction site. According to Tolleth, 
three measurements must be correct to achieve a proper placement of the auricle; AXIS, LEVEL and DISTANCE from the orbit. [5]

Axis: It is difficult to define exactly the positioning of the axis, but it can be described as the "line of balance" through the long dimension of the ear. Some indicate that axis is parallel to the bridge of nose. An angulation of 20 degree from vertical position seems to be satisfactory.

Level: The level can be assessed with the head in the anatomic vertical position. The highest part of the helix is on a line roughly with that of the eyebrow, and the lowest part of the lobule is on a line at the base of columella or slightly below that.

Distance from the orbit: The ideal distance of the prosthesis from the lateral orbital rim is about one ear length, or 6.5 to $7.5 \mathrm{~cm}$.

\section{Retention of the prosthesis}

The retention and stability of the prosthesis is an important factor for the prosthesis; hence the ear prosthesis can be retained by various methods of retention, either by using anatomical undercuts, hair bands, and frame of eyeglasses, adhesives and implants with magnets or bars.[6]

Although implants can provide better retention and stability of the prosthesis, the reported drawback of implants was high number of failure rates due to the effect of radiation therapy on bone morphology, the compromised healing of the skin in the region of the mastoid and accuracy of impression over movable tissues. [7] In addition, cost factor of the implants and the waiting period was not acceptable by the patient.

Another major disadvantage was that due to psychological trauma of undergoing oncosurgery, the patient hardly agrees to undergo another surgery for

Implant placement. Thus, due to these factors clinicians had no better option rather than using custom made prosthesisfor such patients. [8]

The skin adhesive may degrade and results in reduced strength and bonding property over a long period of time; some skin adhesives have been reported to cause hypersensitive reactions. [8] Although the success rate of implant supported

Prosthesis is very high, the prosthesis retained with skin adhesives, anatomical and soft tissue undercuts are more successful due to their ease of application and are comparatively less expensive then implant supported prosthesis. [9]

Silicone elastomeric materials are more commonly used, because they provide better stability and good marginal adaptation, which satisfies patient's cosmetic and esthetic needs; but the major disadvantage is that the manipulation of silicone requires more complex, advanced and multifaceted techniques which are rather more expensive.[10] The silicone elastomeric material possess' excellent physical properties with good heat stability and are chemically inert materials, particularly when they are used in fabrication of prosthesis used to restore body parts.

Silicon elastomeric material possesses soft tissue like consistency; provide additional advantage when they are used to restore the defects in movable soft tissues. Silicon materials are available in various shades provided by manufacturers to give exact shade and texture of skin which closely simulate and resemble shade of patient's skin complexion. The drawback of the silicon prosthesis is that, in the long term the prosthesis material degrades easily and its additives undergo changes when exposed to moisture, high temperature, UV light and sunlight, thus creating a need for replacement by a new prosthesis. To overcome these disadvantages newer polymeric materials have been introduced like polyphopozenes, silicon block polymers, methacryloxypropyl terminated polydimethylsiloxane with enhanced mechanical, chemical and physical properties, such as increased elongation, high edge strength, improved heat stability, good tear strength, chemically inert, low hardness and viscosity for fabrication of maxillofacial prostheses. [8]

In our current case, the ear prosthesis was replicated well 
and bore good resemblance to the patient's right side ear from the frontal and rear views. The colour matching with intrinsic shades were a close match but they were improved with help of extrinsic stains. The prosthesis was fitted with the help of medical grade adhesive and the anatomical undercuts available from the remnants of the auricle on the left side. The patient was hesitant to wear spectacles for the retention of the prosthesis. The anterior margins were strategically hidden by the hairline while the posterior borders merged well in shade to the skin. Overall,

\section{References}

1. G. G. G. R. P. R. David F. Butler, "Silicone auricular prosthesis," J Am Acad Dermatol , vol. 43, pp. 687-90, 2000.

2. K. R. K. S. B. S. M. H. Bandlar H. Naveen, "A Blue Tooth - Auricular Prosthesis: A Case Report," J Clin Diagn Res. , vol. 8, no. 8, pp. 36-38, 2014.

3. S. N. Madhan R, "Prosthetic management of patient with treacher collins syndrome," Ind J Dent Res, vol. 17, no. 2, 2006.

4. M. E. B. G. B. K. Y. Gunay, "Facilitation of facial prosthesis placement with tattoo markers: A clinical report," J Prosthet Dent, vol. 97, pp. 265-260, 2007.

5. M. Ferraz DeOliveira, Auricular prosthesis. Osseointegration in craniofacial reconstruction., Chicago: Quintessence, 1998, pp. 213221.

6. A.S.C.S. Robin W. C. Chung, "Magnet-retained auricular prosthesis with an implant-supported composite," J Prosthet Dent, vol. 89, pp. 446-9, 2003.

7. J. D. A. Todd M. Kubon, "An implant-retained auricular impression technique to minimize soft tissue," J Prosthet Dent, vol. 89, pp. 97$101,2003$.

8. M. K. P. D. S. K. N. Padmanabhan T, "Prosthetic rehabilitation of orbital and facial defect," Journal of Prosthodontics., vol. 20, p. 200, 2012.

9. M. R. J. P. Somkuwar K, "prosthesis: Patient rehabilitation- a case report," People's J ournal of Scientific research, vol. 2, no. 2, pp. 21-26, 2009.

10. W. M. J. R. Aziza T, "Analysis of the properties of silicone rubber maxillofacial prosthetic materials.," Journal of Dentistry, vol. 31, pp. $67-74,2003$. the patient expressed complete satisfaction in the auricular prosthesis.

\section{Conclusion}

Difficulties encountered during the reconstructive process. It is difficult to simulate the exact anatomy of the contra lateral side. But the smile on the patient face after the reconstruction gives satisfaction and encouragement to help people live better with the little services that we can provide. 